\title{
Bibliometría aplicada a la gamificación como estrategia digital de aprendizaje ${ }^{1}$
}

\author{
Bibliometrics applied to gamification as a digital learning \\ strategy \\ José Peirats Chacón \\ Universitat de València, València, España \\ jose.peirats@uv.es \\ Diana Marín Suelves \\ Universitat de València, València, España \\ diana.marin@uv.es \\ $\mathrm{M}^{\mathrm{a}}$ Isabel Vidal Esteve \\ Universitat de València, València, España \\ Ivies7@uv.es
}

\begin{abstract}
Resumen
Este trabajo se interesa por un tema de actualidad como es la gamificación en educación, una interesante estrategia didáctica para responder a los alarmantes datos sobre fracaso y abandono escolar obtenidos año tras año en las pruebas diagnósticas realizadas en el ámbito europeo. A través del estudio bibliométrico de la producción científica se ha analizado las principales tendencias en gamificación, como práctica mediada por la tecnología en las aulas de las diferentes etapas educativas, a través de 137 documentos alojados en la base de datos Scopus y publicados en la última década. En los resultados se revela que el número de documentos se ha incrementado notablemente en los últimos años; sin embargo, el impacto en las prácticas digitalizadas de aula sigue siendo escaso, exceptuando algunos casos. Se destaca que el peso del continente europeo es notable en este campo, si lo comparamos con el americano o el asiático. Y también que la mayoría de las publicaciones se sitúan en Primaria, existe un cierto equilibrio entre asignaturas científicas y humanísticas y los dispositivos de preferencia son las tabletas. Concluimos apuntando contrastar sistemáticamente estudios por etapas y reflexionar sobre las estrategias educativas en el contexto de la sociedad digital.
\end{abstract}

Palabras clave: gamificación, estrategia educativa, aprendizaje, base de datos, tecnología.

\begin{abstract}
This work is interested in gamification education, an interesting didactic strategy to respond to the alarming data on school failure and dropout obtained year after year in diagnostic tests carried out in Europe. Through the bibliometric study of scientific production, the main trends in gamification have been analysed, as a practice mediated by technology in the classrooms of the different educational stages, through 137 documents included in the Scopus database and published in the last decade. The results reveal that the number of documents has increased significantly in recent years; however, the impact on digitised classroom practices is still limited except in a few cases. It should be noted that the weight of the European continent is remarkable in this field, if we compare it with the Americas or Asia. And also that most of the publications are located in Primary, there is a certain balance between scientific and humanistic subjects and preferred devices are tablets. We conclude by systematically contrasting studies by stages and discussing educational strategies in the context of digital society.
\end{abstract}

Key words: gamification, educational strategy, learning, database, technology.

\footnotetext{
${ }^{1}$ Los autores de este estudio pertenecen al Grupo de Investigación CRIE (GIUV2013-105) de la Universitat de València.
} 
RED. Revista de Educación a Distancia. Núm. 60, Artíc. 05, 30-10-2019

DOI: http://dx.doi.org/10.6018/red/60/05

\begin{abstract}
Sommario
Questo documento si interessa di gamification in materia di istruzione, un interessante strategia didattica per rispondere ai dati attuali molto allarmanti sul fallimento e l'abbandono scolastico, ottenuti anno dopo anno nei test diagnostici condotti in Europa. Attraverso lo studio bibliometrico della produzione scientifica, sono state analizzate le principali tendenze dell'uso della gamification, come pratica mediata dalla tecnologia nelle aule dei diversi ordini scolastici, attraverso 137 documenti presenti nel database Scopus e pubblicati nell'ultimo decennio. I risultati rivelano che il numero di documenti è aumentato significativamente negli ultimi anni; tuttavia, l'impatto sulle pratiche di classe digitalizzate è ancora limitato, tranne in alcuni casi. In particolare, si sottolinea che il peso del continente europeo è notevole in questo campo, se lo confrontiamo con quello americano o asiatico. Inoltre, la maggior parte delle pubblicazioni sono riferite alla scuola primaria, c'è un certo equilibrio tra materie scientifiche e umanistiche e i dispositivi preferiti sono i tablet. Concludiamo sistematicamente confrontando gli studi per fasi e riflettendo sulle strategie educative nel contesto della società digitale.
\end{abstract}

Parole chiave: gamification, strategia educative, apprendimento, database, tecnologia.

\title{
1. Introducción
}

España es uno de los países de la Unión Europea peor situados en cuanto a fracaso y abandono escolar. Según los datos más recientes obtenidos por el Instituto Nacional de Estadística (MEFP, 2018), un 18,3\% de los estudiantes europeos que abandonan el sistema tempranamente son españoles, solo por debajo de los malteses con $18,6 \%$. En esta misma línea, un reciente estudio (Pérez \& Aldás, 2019) centrado en la situación en las universidades españolas señala la elevada tasa de abandono de los grados de diferentes ramas de conocimiento y de los estudios universitarios en general, que supera el $30 \%$ en la cohorte analizada. Además, en este estudio se hace referencia a los déficits en los planes de estudios, a la baja calidad de la docencia impartida o a la falta de vocación, como motivos principales del abandono. En contraposición, tal y como apuntan varios estudios (García \& Jiménez, 2019; Panadero, 2019), el motivo principal del fracaso escolar, después de los problemas en autoconcepto y autoestima, es la falta de ilusión, motivación y entusiasmo por el aprendizaje. Estos datos nos llevan a plantearnos la necesidad de realizar propuestas de estrategias innovadoras que aborden estos problemas, basadas en los intereses y competencias del alumnado.

Es evidente que, desde hace ya tiempo, se están introduciendo cambios en los centros educativos producto del proceso de digitalización global, y que se ven reflejados en un aumento de dotación tecnológica o en una mayor oferta formativa para el profesorado relacionada con su implementación en las prácticas educativas (Marín \& Vidal, 2019). A pesar del preocupante análisis de la realidad del que se hacen eco investigadores y medios de comunicación, y de los esfuerzos de la Administración, profesionales de la educación y empresas editoriales por adaptarse a los cambios de la sociedad del siglo XXI, las prácticas de aula parecen estar todavía ancladas en tiempos pasados y el uso de los recursos tecnológicos disponibles en los centros educativos queda, en demasiadas ocasiones, relegado a actividades esporádicas realizadas bajo el criterio arbitrario del profesorado (Henao, Ballesteros \& Holguín, 2019) o en un cambio de soporte que no se acompaña de mejoras sustantivas en educación. Sin embargo, el uso de los materiales didácticos digitales tiene importantes implicaciones en las prácticas de enseñanza y aprendizaje (Area \& González, 2015) y la tecnología abre un mundo de posibilidades para la mejora de la calidad de la enseñanza a través de la adaptación al alumnado del siglo XXI y a sus características y necesidades.

El impacto de las tecnologías en la educación es innegable y reflejo de esta realidad son las estrategias didáctico-digitales que el profesorado está implementando en los últimos tiempos en las aulas de las diferentes etapas educativas. Ejemplo de ello,

Bibliometría aplicada a la gamificación como estrategia digital de aprendizaje. J. Peirats, D. Marín y M.I. Vidal

Página 2 de 26 
son las metodologías activas que conllevan la puesta en práctica de estrategias como el aula invertida, el aprendizaje cooperativo en red o la gamificación, entre otras.

Fijándonos en la última como muestra, si bien es cierto que se ha avanzado en el uso de la gamificación en el sistema escolar en estos últimos años también lo es que, hasta la fecha, a tenor de algunos autores, se ha publicado poco al respecto, apenas algunas anécdotas sobre su uso en situaciones educativas concretas en algunas investigaciones que abarcan principalmente las percepciones de los estudiantes universitarios (Gooch, Vasalou \& Benton, 2016). Sin embargo, afirmaciones de ese calibre merecen comprobarse nuevamente y por este motivo, se considera necesario seguir indagando en este campo, para profundizar en las evidencias basadas en la práctica y al establecimiento de pautas de actuación compartidas que conduzcan a experiencias de éxito.

En el tema que nos ocupa en este estudio, coincidimos en que la base del aprendizaje es el juego, pero esta idea no es nueva. Autores como Piaget (1956), Schiller (1945) y Huizinga (1968) entienden el juego como un reflejo de las estructuras cognitivas existentes y a su vez como el medio para alcanzar nuevos aprendizajes. Pero si avanzamos en el tiempo y consideramos, en el tema del juego, a los actuales nativos digitales (Prensky, 2010), hijos de la sociedad red, de la sociedad digital o de la denominada sociedad del conocimiento, es preciso detenerse ante las prácticas de aprendizaje mediadas por tecnologías basadas en el juego, lo que se conoce ahora como gamificación o ludificación.

La gamificación es un término relativamente reciente que se define, según Kapp (2012), como la utilización de mecanismos del juego, la estética y el uso del pensamiento para atraer a las personas, incitar a la acción, promover el aprendizaje y resolver problemas; para Zichermann y Cunnigham (2011), como el proceso relacionado entre el pensamiento del jugador y las técnicas de juego para atraer a los usuarios y resolver problemas; y considerando a Werbach y Hunter (2012), consiste en la utilización de estructuras, características, dinámicas y estéticas del juego en ámbitos cuya naturaleza no es lúdica.

Dicho esto, es necesario remarcar que todos hacen hincapié en que se trata del uso de las experiencias lúdicas, en ambientes no lúdicos, con el fin de modificar comportamientos humanos aprovechando el componente motivacional. Es de destacar que, a diferencia de los videojuegos o del juego propiamente dicho, el objetivo principal de la gamificación es el de influir en la conducta psicológica y social de las personas a través de retos y/o incentivos (Przybylski, Rigby \& Ryan 2010; Vassileva, 2012), independientemente de otros objetivos secundarios como pueden ser las experiencias hedonistas (Díaz \& Troyano, 2013). Además, se busca involucrar al jugador en tareas atractivas y entretenidas a través de la creación de ambientes y espacios atrayentes y de motivación, para aumentar la participación de los usuarios (Flatla, Gutwin, Nacke, Bateman \& Mandryk, 2011). Para conseguir sus objetivos se basa en el uso de sus tres elementos básicos: dinámicas -como estructura implícita del juego-, mecánicas -como procesos que lo desarrollan- y componentes -como implementaciones específicas: avatares, insignias, ránquines, etc.- (Werbach \& Hunter 2012).

Por su parte, ludificación, aunque algunos autores lo consideran la mejor traducción de gamification al español y es la opción correcta en nuestro idioma, según la RAE; el término adquiere un carácter menos pedagógico y se relaciona mayormente con el sentido placentero del propio juego, implica hacer lúdico algo que no es divertido. La palabra lúdico es un adjetivo que califica a aquello relacionado con el juego, y proviene del latín ludus o ludere, cuyo significado es jugar, divertirse, entretenerse; entendido como actividad placentera, donde el ser humano se libera de tensiones y de las reglas impuestas por la cultura (Oliva, 2016). Este segundo término podría definirse como el conjunto de iniciativas que, agregando elementos lúdicos, pretenden motivar a la participación en ambientes no propios para ello (Vivar, 2015).

Bibliometría aplicada a la gamificación como estrategia digital de aprendizaje. J. Peirats, D. Marín y M.I. Vidal

Página 3 de 26 
Partiendo de sus definiciones puede apreciarse que los dos términos presentan ciertos matices, sin embargo, serán tomados en nuestra investigación, tal y como lo hacen otros autores (Martí, 2017; Ortiz \& Suárez, 2018; Sánchez, 2017) como sinónimos, ya que no es nuestro objetivo diferenciarlos, sino analizar la producción científica sobre el tema.

Si la gamificación hasta no hace mucho tiempo se relacionaba con la utilización de elementos del juego dentro de entornos como los negocios o la salud (Raftopoulos, 2014; Zicherman \& Cunningham, 2011), ahora ya está plenamente incorporada en ciertas experiencias aplicadas a los entornos de aprendizaje. Además, según Cortizo et al. (2011), se puede gamificar cualquier proceso o actividad susceptible de ser aprendida, y en este estudio nos vamos a centrar en sus ventajas como una posible estrategia educativa para abordar el conocido fracaso escolar.

En los últimos tiempos mucho se ha escrito sobre qué es la gamificación (Gómez \& García, 2018), cómo y cuándo introducirla en el aula, los beneficios que se alcanzan para el proceso de enseñanza y aprendizaje y sus actores principales, docentes y alumnado. Se han descrito prácticas de gamificación en las diferentes etapas educativas, desde Educación Infantil a la Educación Superior (Corchuelo, 2018; Gómez \& Ruiz, 2017; Pintor, 2017), pasando por la etapa de Educación Primaria (Fernández, Prieto, Alcaraz, Sánchez \& Grimaldi, 2018; Gaviria, Gallego, Rodríguez, Oliver \& Puyana, 2018; Martínez, 2017), y por la Educación Secundaria Obligatoria (Manzano \& Baeza, 2018), y en diferentes tipos de escuelas como puede ser en la escuela rural (Alamanda et al., 2019; Bona, 2016; Botha \& Herselman, 2015).

Entre los diferentes resultados obtenidos de ciertas investigaciones recientes, se describen los efectos positivos sobre el aprendizaje y el alumnado en general, como pueden ser el incremento de la motivación (Hassan, Dias \& Hamari, 2019), el desarrollo de la personalidad (Ghaban \& Hendley, 2019) y del pensamiento reflexivo (Peña, Fernández, Kirillof \& Tovar, 2011), el interés por el aprendizaje y la participación activa (Lee and Hammer, 2011; Measles \& Abu-Dawood, 2015; Rouse, 2013) y la adquisición de aprendizajes curriculares (Gros, 2008); los hábitos de aprendizaje y las habilidades para la resolución de problemas (Harrold, 2015), la mejora de la socialización (Montilla, 2015; Park \& Kim, 2019), el trabajo cooperativo y la comunicación entre iguales (Roemer \& Rundle, 2018; Sánchez, Alfageme \& Serrano, 2010), el desarrollo de reflejos, psicomotricidad (Rodríguez \& Martín), iniciativa y autonomía e identidad en niños y jóvenes (Aguilar \& Aguilar, 2018), la participación de las familias en el proceso educativo (Vázquez \& López, 2016), o el de adquirir competencias tecnológicas: cognitivas, metodológicas y organizativas (Borrás, 2018; Fernández, Rodríguez \& Verdeja, 2017).

Muy loable es considerar el registro de cuantiosos beneficios para el alumnado con necesidades específicas de apoyo educativo; en particular, por el desarrollo de la creatividad (Ballesta \& Lozano, 2007), la competencia intelectual (Lozano, Castillo \& Veas, 2014), la fortaleza emocional, la estabilidad y el sentimiento de estar a gusto (Rapp et al., 2018) y también por la plasticidad, toma de decisiones, memoria, concentración (Labrador \& Villegas, 2016), capacidad de superación y de control sobre sus propios actos (Kim, Song, Lockee \& Burton, 2017). Además, destacamos que favorece a la actuación de este alumnado; es decir, contribuye a convertir la participación en acción (Jiménez \& García, 2015; López, Vidal, Peirats \& San Martín, 2017); y, también, colabora en la equidad, favoreciendo el acceso a los contenidos por parte de las personas con mayores dificultades para poder hacerlo (López, Vidal, Peirats \& Peirats, 2017; Smith \& Abrams, 2019). Finalmente, como recoge Barros (2016), esta estrategia didáctica se considera muy ventajosa porque contribuye a la asociación entre el aprendizaje y el disfrute.

Antes de dar paso al siguiente apartado, es necesario diferenciar y contextualizar varios términos que suelen asociarse a los de gamificación (o ludificación). Por una parte, el término Edutainment es el concepto general bajo el que se contemplarían estas

Bibliometría aplicada a la gamificación como estrategia digital de aprendizaje. J. Peirats, D. Marín y M.I. Vidal 
prácticas y se refiere al conjunto de contenidos creados especialmente para combinar el entretenimiento y el aprendizaje al mismo tiempo (Becker 2016; Gros, 2008; Soto, Vivancos \& Ferrer, 2018). Por otra parte, Serious Games, se refiere a aquellos juegos (con o sin dispositivo digital) que adquieren un carácter formativo, es decir, que se emplean con propósitos didácticos, más allá del disfrute lúdico, y que suelen estar complementados por técnicas gamificadas específicas, como los niveles, las puntuaciones o las bonificaciones (Girard, Ecalle \& Magnan, 2013), además pueden brindar la oportunidad de participar en simulaciones lúdicas a través de narrativas que influyen en las experiencias de las personas (Marsh, 2011). La diferencia principal con la gamificación radica en que esta no es un juego en sí mismo, como los serious games, sino que se refiere al conjunto de estrategias que se emplean para gamificar un juego (Ordás, 2016). Por su parte, Biel y García (2015) lo resumen con otro matiz; afirman que los serious games buscan el aprendizaje a través del juego, el edutainment es aquello que permite jugar a través del aprendizaje y la gamificación es el uso de los elementos de juego en un contexto no lúdico.

Sin embargo, y pese a todo lo expuesto, no hay que dejar de considerar que el hecho de desarrollar este tipo de estrategias en las aulas puede ser contraproducente puesto que no todo son ventajas: el coste de la creación de los materiales es elevado, requiere de mucho tiempo de utilización para lograr mejoras en el aprendizaje, puede incrementar la distracción en el alumnado y requiere encontrar el punto de equilibro entre el juego en sí y la transferencia de aprendizajes (Martín \& Víchez, 2017; Tori, 2016). Estas problemáticas habrá que tenerlas en cuenta y, además, será muy importante establecer relaciones entre los retos que se presentan al alumnado y sus capacidades para llevarlas a cabo con el fin de mantener, de nuevo, el equilibrio entre el desafío y la dificultad; un reto demasiado fácil provocará aburrimiento y uno inalcanzable supondrá frustración, concluyendo ambas opciones en la pérdida del objetivo principal, la motivación por el aprendizaje (Castellón \& Jaramillo, 2012; Ortiz, Jordán \& Agredal, 2019).

De acuerdo con lo anterior, es fundamental que los docentes tengan en cuenta una serie de elementos considerados por varios autores (Corchuelo, 2018; Ramírez, 2014; Rodríguez \& Santiago, 2015; Werbach \& Hunter, 2012) como necesarios para implementar estrategias gamificadas de forma exitosa, lo que conllevaría ventajas, según losup y Epema (2014), como la mejora en las habilidades para motivar a su alumnado a través del uso de distintas estrategias y dinámicas:

1. Definir con claridad los objetivos a conseguir para diseñar estrategias eficaces y coherentes.

2. Delimitar los comportamientos a potenciar en el alumnado (conocimientos, actitudes, habilidades, etc.)

3. Establecer los rasgos y las características de los jugadores para diseñar actividades pertinentes a sus intereses y a sus capacidades.

4. Establecer los ciclos de las actividades definiendo el sistema mediante mecánicas de juego, interacciones, orden de los eventos, etc.

5. Describir los eventos para la diversión, como base del juego.

6. Incluir todas las herramientas y recursos a emplear para el desarrollo de la estrategia.

Acabando este apartado señalamos que, con este estudio, se ha pretendido revisar el estado del arte sobre un tema de elevada actualidad y gran impacto en la juventud, empleando para ello un estudio bibliométrico centrado en la implementación de la gamificación en educación. La relevancia de esta cuestión se justifica por el elevado impacto que la implementación de lo digital está teniendo en el ámbito educativo (Area, 2018), por la preocupación por redefinir el rol de los agentes educativos en este cambio de paradigma (San Martín \& Peirats, 2018) y por las posibilidades que parece

Bibliometría aplicada a la gamificación como estrategia digital de aprendizaje. J. Peirats, D. Marín y M.I. Vidal

Página 5 de 26 
ofrecer la gamificación como estrategia metodológica para la mejora de la calidad de la enseñanza (López, Avello, Baute \& Vidal, 2018). Además, este trabajo permite presentar una serie de beneficios alcanzados como estrategia educativa en diferentes etapas educativas, como pueden ser el contribuir al aumento del rendimiento académico y a la reducción del absentismo y el fracaso escolar. Para conseguirlo los objetivos planteados en el estudio han sido:

- Describir las principales características de las investigaciones realizadas en la última década sobre la gamificación en educación.

- Analizar el contenido de las publicaciones para sistematizar el conocimiento científico generado sobre el uso de la tecnología y la gamificación.

\section{Metodología}

Desde una perspectiva cienciométrica se ha realizado este estudio bibliométrico, un enfoque que consiste en analizar los rasgos cuantificables de la literatura científica y que nos permite aproximarnos objetivamente a las diferentes dimensiones que componen la realidad científica de un campo de estudio concreto (Tomás-Górriz \& Tomás-Casterá, 2018), en este caso la gamificación educativa. Los indicadores bibliométricos son válidos en aquellas áreas en las que las publicaciones son un resultado esencial de la investigación y, por esta razón, su validez es máxima en el estudio de las grandes áreas donde predomina la publicación científica, como es el caso. Además, siguiendo las consideraciones de Bordons y Zulueta (1999) sobre la fiabilidad de los estudios bibliométricos, según el tamaño de las unidades o del área de estudio, se ha considerado conveniente añadir un mesoanálisis, con el propósito de analizar la producción sobre la temática en un área científica concreta: las Ciencias Sociales (King, 1978).

Partiendo de la premisa de que las bases de datos bibliográficas son la principal fuente de información que se utiliza en los estudios bibliométricos y que la validez de un estudio dependerá en gran medida de que la base de datos seleccionada cubra de forma adecuada el área objeto de estudio, se ha considerado pertinente investigar en la conocida base Scopus, pues ha sido considerada por varios autores (Granda et al., 2013; Hernández, Sans, Jové \& Reverter, 2016) como la más completa en cobertura temporal y en número de títulos por área.

En la realización de este estudio se diferencian cinco fases, cuatro de ellas correspondientes al análisis bibliométrico y una complementaria centrada en el contenido de los documentos. La primera de ellas se centra en el establecimiento de la ecuación de búsqueda, que viene a ser la combinación de las palabras clave empleadas. La segunda consiste en la selección de los documentos científicos para el análisis, tras una lectura inicial de los resúmenes. La tercera fase implica el análisis de la información recopilada y la cuarta, la emergencia de hallazgos. La última fase, consiste en un análisis de contenido para profundizar en el estado de la cuestión en el área de las Ciencias Sociales.

La muestra final está compuesta por el total de documentos obtenidos tras la búsqueda en dicha base de datos mediante las palabras clave: gamification OR Iudification OR edutainment AND education AND primary OR nursery AND teaching OR learning aparecidos entre el año 2008 y el $2018(n=137)$. Sobre ella, se llevó a cabo el análisis bibliométrico de las 137 publicaciones obtenidas en la primera búsqueda. Posteriormente, se llevó a cabo una selección de los documentos pertenecientes al área de las Ciencias Sociales, resultando un total de 59 documentos, que han sido objeto del análisis de contenido. En este segundo análisis se emplearon fichas de registros (ver Tabla 1) para recoger información referente a las distintas etapas educativas, asignaturas, dispositivos y aplicaciones empleados, así como los principales resultados de la investigación.

Bibliometría aplicada a la gamificación como estrategia digital de aprendizaje. J. Peirats, D. Marín y M.I. Vidal 
RED. Revista de Educación a Distancia. Núm. 60, Artíc. 05, 30-10-2019

DOI: http://dx.doi.org/10.6018/red/60/05

Tabla 1

Indicadores de la ficha para el análisis de contenido de los documentos de Ciencias Sociales

\begin{tabular}{|c|c|c|c|c|c|}
\hline \multicolumn{6}{|c|}{ Ficha de registro de contenido } \\
\hline Artículo & $\begin{array}{l}\text { Etapa } \\
\text { educativa }\end{array}$ & Asignatura & $\begin{array}{l}\text { Dispositivo } \\
\text { empleado }\end{array}$ & $\begin{array}{l}\text { Aplicación } \\
\text { empleada }\end{array}$ & Resultados \\
\hline
\end{tabular}

Fuente: Elaboración propia.

Para el análisis de datos se ha empleado también la herramienta Vosviewer (Van Eck \& Waltman, 2010) con el fin de efectuar la construcción y representación gráfica de un mapa de coocurrencia de términos, con conteo fraccional, ya que ha demostrado ser preferible al completo (Perianes, Waltman \& Van Eck, 2016). Además, se han establecido una serie de indicadores de colaboración científica de primera generación, basándonos en los propuestos por Aleixandre (2010), que se agrupan en torno a la productividad científica, la colaboración, la repercusión y el impacto. Por último, se han tenido en cuenta las variables estructurales y de contenido en base a las categorías que han ido emergiendo en la lectura de los textos.

\section{Resultados}

En cuanto a la productividad científica se analiza, en primer lugar, el número de documentos publicados por año, sin limitar el periodo temporal inicial, con el fin de estudiar la frecuencia de publicación en este tema, conocer sus inicios en el ámbito de la investigación, así como la tendencia general diacrónica. El periodo temporal del que se han obtenido resultados es de catorce años, entre el 2004 y el 2018, y el número de trabajos obtenidos fue de 137, tal y como se ha comentado anteriormente.

En la Figura 1 se puede comprobar que, siguiendo la línea de tendencia de la media móvil, especialmente empleada para calcular las tendencias en largos periodos temporales, el número de publicaciones ha ido incrementándose a lo largo de los años, pero no de forma uniforme, hasta el período final (de 2014 a 2018). El análisis de los datos muestra que en los primeros años se registran muy pocos documentos, y que el contenido de la mayoría de ellos no se refiere a la gamificación entendida como tal. Como ejemplo, el primer documento, el de Sugimoto, Kusunoki, Inagaki, Takatoki y Yoshikawa (2004), detalla una intervención a través de un sistema de apoyo al aprendizaje colaborativo mediante paneles de detección basados en tecnología RFID, lo que no implica una intervención gamificada. Lo mismo sucede con los publicados en 2006 y 2007 (Gloria, Margarone \& Graco; 2007; Jesse, 2006, Liu, Cheok, Mei-Ling \& Theng 2007), que se refieren al empleo de la robótica, realidad virtual y los cursos de informática basados en lerning-by-doing en centros educativos, respectivamente. A partir de 2013 ya se registra un incremento notable en las publicaciones, hasta llegar a 35 documentos en el 2018. Esta información confirma la presentada en el informe Horizon 2014 (Johnson, Adams, Estrada \& Freeman, 2014), en el que se pronosticaba la gamificación como una de las estrategias metodológicas que en un período de tres años calaría con fuerza en el ámbito educativo.

Bibliometría aplicada a la gamificación como estrategia digital de aprendizaje. J. Peirats, D. Marín y M.I. Vidal

Página 7 de 26 


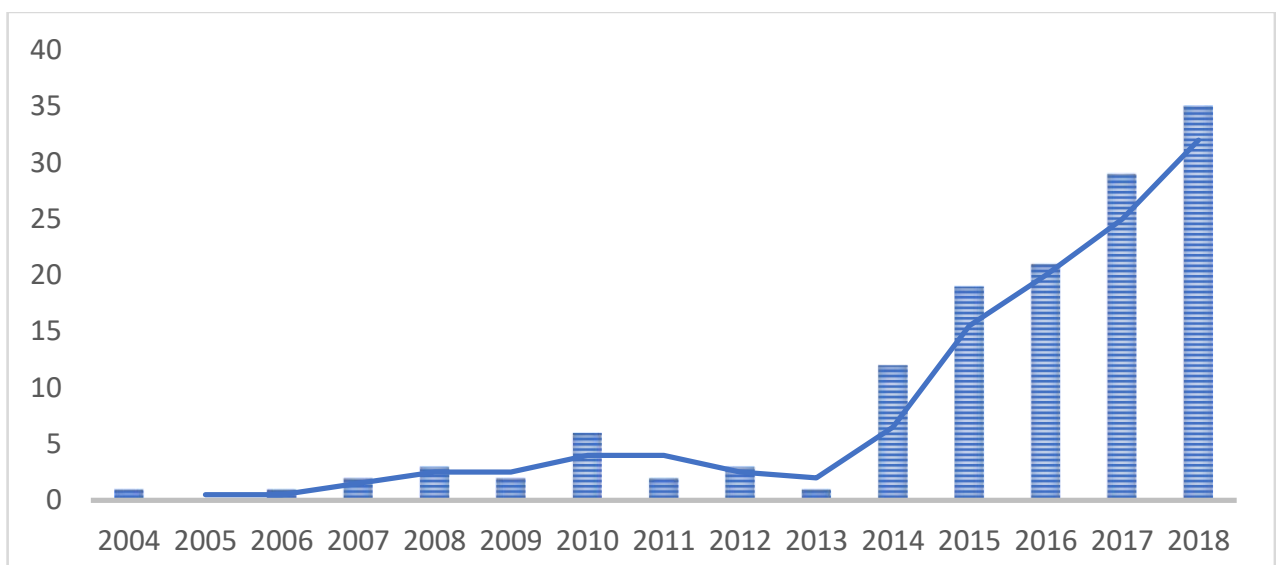

Figura 1. Frecuencia anual de publicaciones en Scopus y tendencia. Fuente: Elaboración propia.

Por otra parte, en la Figura 2 se procede a analizar el tamaño bibliométrico de cada uno de los países en los que se registran publicaciones sobre el tema durante el periodo estudiado.

Existe un claro desequilibrio ya que destacan una serie de países, entre ellos dos: Italia y España, con dieciséis y catorce documentos respectivamente. A los que les siguen Grecia y Reino Unido con once artículos y Alemania y Malasia con siete registros cada uno. Quedan, además, un grupo de países con menor representación, entre tres y cinco documentos, como son Brasil, Sri Lanka o Estados Unidos, entre otros. Es de destacar que cinco de los seis países punteros pertenezcan a Europa, y además a la zona suroeste; lo que sitúa a nuestro continente como una de las áreas más productivas del mundo respecto a la temática. Lo que coincide con el análisis de Ovallos, Villalobos, De la Hoz y Maldonado (2016), que muestra una tendencia creciente del impacto del concepto de gamificación en las revistas científicas desde el año 2010 y un bajo nivel de producción científica sobre este tema en lugares como Latinoamérica.

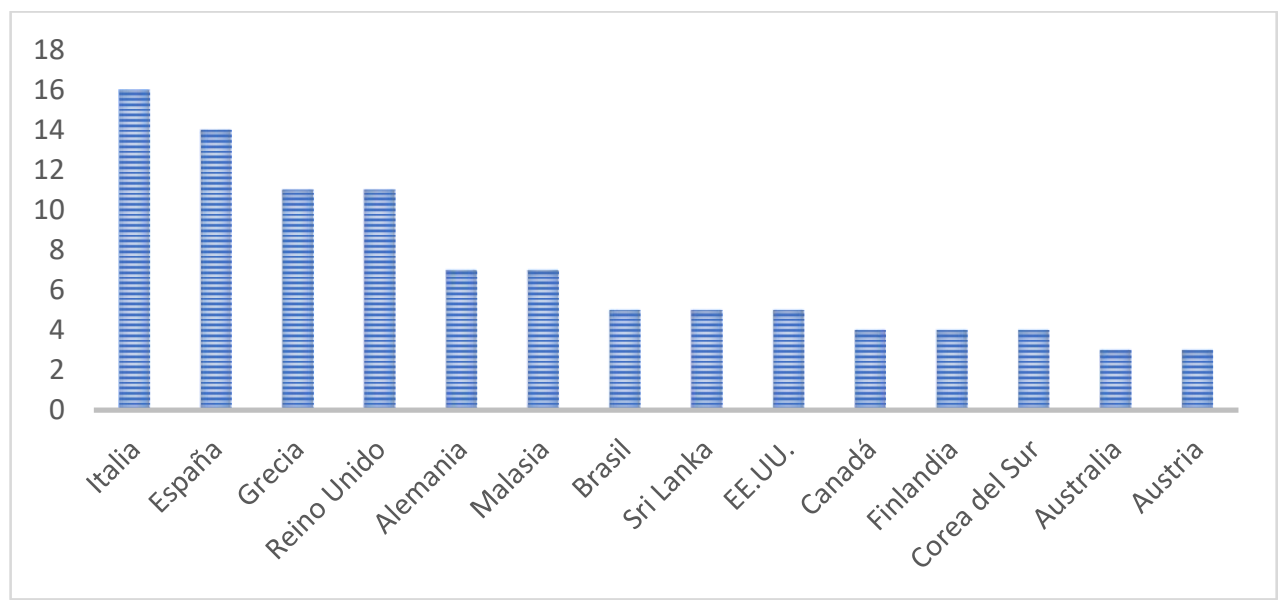

Figura 2. Número de publicaciones por país. Fuente: Elaboración propia.

Relacionado con el análisis por países, se ha considerado subrayar las instituciones más prolíficas en el ámbito, donde despuntan universidades españolas-con publicaciones muy actuales. Siguiendo a las universidades foráneas de Bozen, Bolzano, y Colombo, con siete las dos primeras y cuatro documentos publicados la tercera, destaca la Universidad de Extremadura con tres artículos (Marín, López \& Maldonado, 2015; Sánchez, Cañada \& Dávila, 2017; Sánchez, Cañada \& Dávila, 2018). Le acompañan las universidades Politècnica de València, con dos documentos (García, 
Jurdi, Jaén \& Nácher, 2018; Pérez, Contero \& Alcañiz, 2018), y, con un artículo cada una, las universidades de Santiago de Compostela (Caldeiro, Yot \& Castro, 2018), Castilla La Mancha (Cózar \& Sáez, 2016), Granada (Pérez, Rivera \& Delgado, 2017), Rey Juan Carlos (García \& Hijón, 2018) y Cádiz (Calderón, Trinidad, Ruiz \& O’Connor, 2018), entre otras.

Si tomamos ahora los datos extraídos de los distintos documentos en cuanto a colaboración y coautoría, en la Tabla 2 se analizan el número de autores para conocer la información concreta acerca de las estructuras y redes de colaboración en la comunidad científica, y con ello el carácter multidisciplinar (en mayor o menor medida) de las publicaciones estudiadas.

Tabla 2

Colaboración y coautoría

\begin{tabular}{lcc}
\hline $\begin{array}{l}\text { Número } \\
\text { autores }\end{array}$ & de & N \\
\hline Uno & 11 & $\%$ \\
\hline Dos & 32 & $8 \%$ \\
\hline Tres & 29 & $23 \%$ \\
\hline Cuatro & 32 & $21 \%$ \\
\hline Cinco & 11 & $23 \%$ \\
\hline Seis & 5 & $8 \%$ \\
\hline Siete & 5 & $4 \%$ \\
\hline Más de 7 & 3 & $4 \%$ \\
\hline AAVV & 9 & $2 \%$ \\
\hline
\end{tabular}

Fuente: Elaboración propia

Los resultados muestran que la mayoría de los trabajos fueron escritos en colaboración (el 85\%). Por su parte, 56 artículos, un 41\% del total, fueron escritos por cuatro o más autores, lo que supone un alto grado de coautoría en este ámbito. Entre ellos destacan el de Rosi, Brighenti et al. (2016) sobre el aprendizaje a través del juego (con nueve autores), el de Rosi, Dall'Asta et al. (2016), sobre el uso de tecnologías en primaria (con diez autores), ambos para la intervención en educación nutricional; $y$ el de Agoritsas et al. (2014) sobre intervenciones múltiples mediante gamificación en estudiantes de medicina (con 13 coautores).

Posteriormente, tras el análisis de los autores firmantes de cada documento, se identifican un total de 155 autores que firman en las 137 publicaciones. Se describe la distribución de la producción en función del número de trabajos encontrados en la base de datos Scopus para un mismo autor y la productividad según la ley de productividad científica de Lotka, que relaciona la cantidad de autores y artículos producidos en un periodo sobre un tema, predominando los productores ocasionales (147) y encontrando seis productores medianos y dos grandes productores, tal y como se observa en la siguiente tabla.

Tabla 3

Bibliometría aplicada a la gamificación como estrategia digital de aprendizaje. J. Peirats, D. Marín y M.I. Vidal

Página 9 de 26 
RED. Revista de Educación a Distancia. Núm. 60, Artíc. 05, 30-10-2019

DOI: http://dx.doi.org/10.6018/red/60/05

Distribución de la producción

\begin{tabular}{cccc}
$\begin{array}{c}\text { No } \\
\text { documentos }\end{array}$ & Autores & $\% \mathbf{n = 1 5 5}$ & Lotka \\
\hline 1 & 122 & $78,70 \%$ & $\begin{array}{c}\text { Productores ocasionales o } \\
\text { pequeños productores }\end{array}$ \\
\hline 2 & 25 & $16,13 \%$ & $\begin{array}{c}\text { Productores ocasionales o } \\
\text { pequeños productores }\end{array}$ \\
\hline 3 & 3 & $1,94 \%$ & Medianos productores \\
\hline 4 & 3 & $1,94 \%$ & Medianos productores \\
\hline 6 & 1 & $0,65 \%$ & Grandes productores \\
\hline 7 & 1 & $0,65 \%$ & Grandes productores
\end{tabular}

Fuente: Elaboración propia

Por tanto, obtenemos que la gran mayoría (el 79\%) de los autores son firmantes de un único documento, por su parte, el $16 \%$ de los autores cuentan con dos artículos. Asimismo, se registran seis autores en total, que corresponden a los medianos productores anteriormente mencionados, tres de ellos son autores de tres documentos (Dodero, Halloluwa \& Vyas) y los otros tres son responsables de cuatro artículos (Hewagamage, Torello \& Usoof). Por último, los dos grandes productores (Melonio \& Gennari) firman conjuntamente en seis de los siete documentos (Di Mascio, Gennari, Melonio \& Torello, 2015; Di Mascio, Gennari, Melonio \& Vittorini, 2013; Dodero, Gennari, Melonio \& Torello, 2015; Dodero, Gennari, Melonio \& Torello, 2014; Gennari, Melonio \& Torello, 2017; Gennari et al., 2017); lo que se ilustra en la figura que sigue.

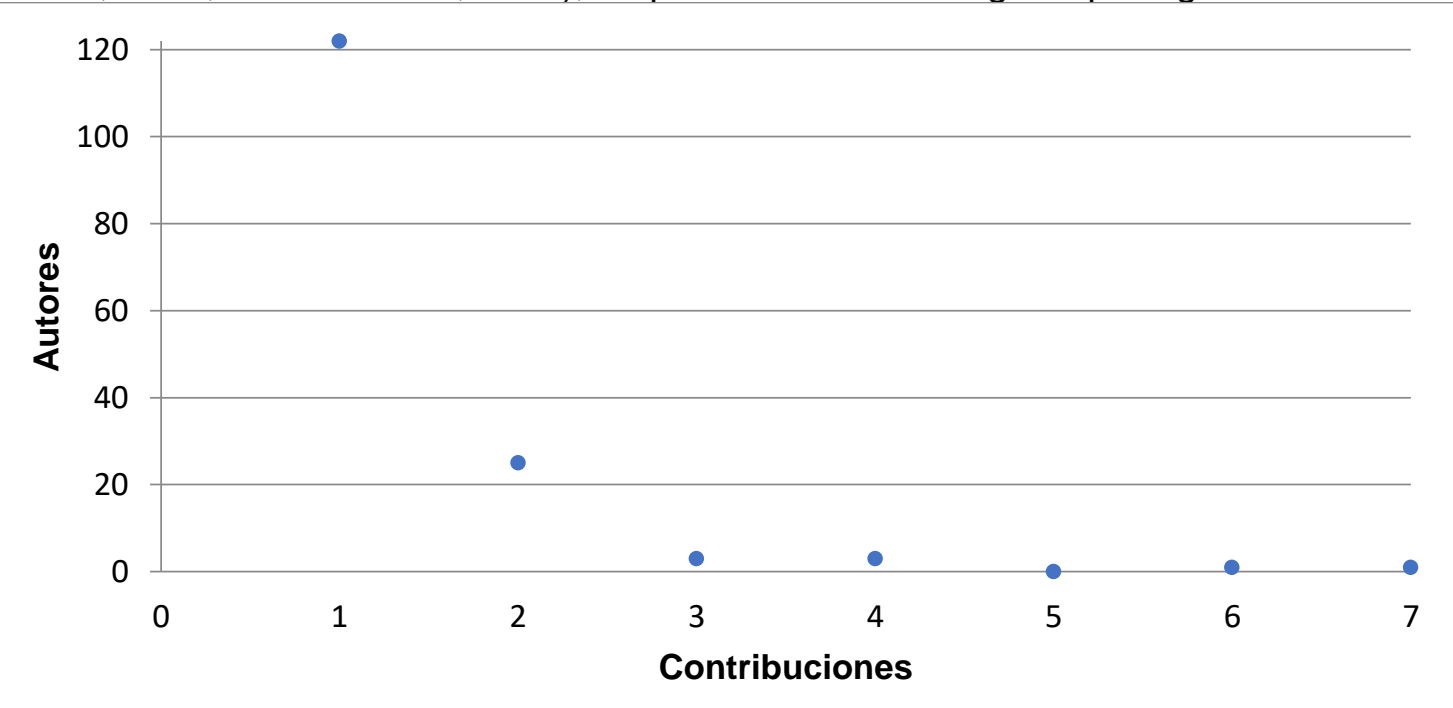

Figura 3. Número de contribuciones por autor. Fuente: Elaboración propia.

Queda exponer que un 7\% de los documentos se corresponden a textos que aparecen en libros de actas y la autoría queda contemplada como: varios autores, por lo que este porcentaje debería sumarse al de los escritos en colaboración, que representan el $92 \%$ de las publicaciones. Si analizamos estos datos de forma longitudinal los resultados no son muy concluyentes ya que se registran artículos de un solo autor tanto en los inicios del período contemplado como en la actualidad, del mismo modo que se observan trabajos con numerosos coautores a lo largo de los distintos años.

Bibliometría aplicada a la gamificación como estrategia digital de aprendizaje. J. Peirats, D. Marín y M.I. Vidal 
Si nos referimos a la repercusión e impacto de los artículos analizados, en la Tabla 4 se puede observar que, del grueso de las publicaciones revisadas, un $53 \%$ no tienen ninguna cita desde su publicación. Lo que podría explicarse en parte, si observamos gráficos anteriores, por ser la mayoría de las publicaciones muy recientes y el lapso de tiempo para poder calcular su impacto corto. Siguiendo con este aspecto, el $31 \%$ conforma el total de artículos que suman entre una y cinco citas, lo que sumaría una gran cantidad trabajos (116) con muy poca repercusión entre la comunidad científica. Sin embargo, es de destacar que cinco documentos cuenten con más de veinte citas, entre los que subrayamos el trabajo de Song y Zhang (2008) que plantea un modelo para el diseño de juegos educativos basado en la conexión entre la motivación y los entornos de aprendizaje efectivos (con 30 citas), y el de los brasileños Da Rocha, Gomes y De Melo (2016), que analizan la eficacia de la gamificación en relación al compromiso del alumnado, con 48 citas pese a su relativamente reciente publicación. Mencionamos, además, el de Caponetto, Earp y Ott (2014) que contiene una revisión bibliográfica sobre gamificación en educación y que cuenta con el mayor registro de citaciones: 56 en solo cuatro años.

Tabla 4

Número de citas recibidas

\begin{tabular}{lcc}
\hline Citas & $\mathbf{N}$ & $\%$ \\
\hline Ninguna & 73 & $53,2 \%$ \\
\hline $1-5$ & 43 & $31,4 \%$ \\
\hline $6-10$ & 6 & $4,4 \%$ \\
\hline $11-15$ & 6 & $4,4 \%$ \\
\hline $16-20$ & 4 & $2,9 \%$ \\
\hline Más de 20 & 5 & $3,6 \%$ \\
\hline
\end{tabular}

Fuente: Elaboración propia

A continuación, se muestran en la Tabla 5, los datos extraídos de Scopus sobre el impacto de citación. Estas cifras muestran la calidad de cita de cada documento, en comparación con otros similares de la misma área, y se consideran variables como el año de publicación, el tipo de documento o las disciplinas, entre otros. Un valor superior a 1 representa que el registro está más citado de lo que se esperaría según su promedio y, como podemos observar, 37 documentos se sitúan en este rango. Si bien es cierto que la mayoría de los documentos cuentan con un índice de impacto nulo o inferior a lo esperado; sin embargo, hay algunos documentos que destacan. Entre aquellos con factor de impacto mayor que 3, señalamos el de Letchumanan, Muthusamy, Govindasamy y Farashaiyan (2016) sobre actividades interactivas en línea con un índice de 8.15; el de Caponetto, Earp y Ott (2014), previamente mencionado, con 8.79 puntos; o el de Souza, Veado, Moreira, Figueiredo y Costa (2017) sobre juegos para el aprendizaje, con 9.35 puntos.

Tabla 5

Índice de impacto

Bibliometría aplicada a la gamificación como estrategia digital de aprendizaje. J. Peirats, D. Marín y M.I. Vidal 
RED. Revista de Educación a Distancia. Núm. 60, Artíc. 05, 30-10-2019

DOI: http://dx.doi.org/10.6018/red/60/05

\begin{tabular}{lcc}
\hline Índice & $\mathbf{N}$ & $\%$ \\
\hline 0 & 81 & $59,1 \%$ \\
\hline $0,1-0,99$ & 21 & $15,3 \%$ \\
\hline 1 & 0 & $0 \%$ \\
\hline $1,01-1,99$ & 13 & $9,4 \%$ \\
\hline $2-2,99$ & 9 & $6,5 \%$ \\
\hline 3 o mayor & 13 & $9,4 \%$
\end{tabular}

Fuente: Elaboración propia a partir de los datos de Scopus.

Como complemento añadimos, también, un análisis que refleja de forma visual la distribución de los documentos obtenidos en la búsqueda, según su tipología. Si observamos el gráfico de la Figura 4, podemos obtener que el 59\%, la mayoría, son comunicaciones de congresos; además, entre ellos se encuentran los documentos con mayor índice de impacto anteriormente destacados. Por su parte, el $27 \%$ son artículos, el $26 \%$ revisiones y, únicamente, el $2 \%$ capítulos de libro.

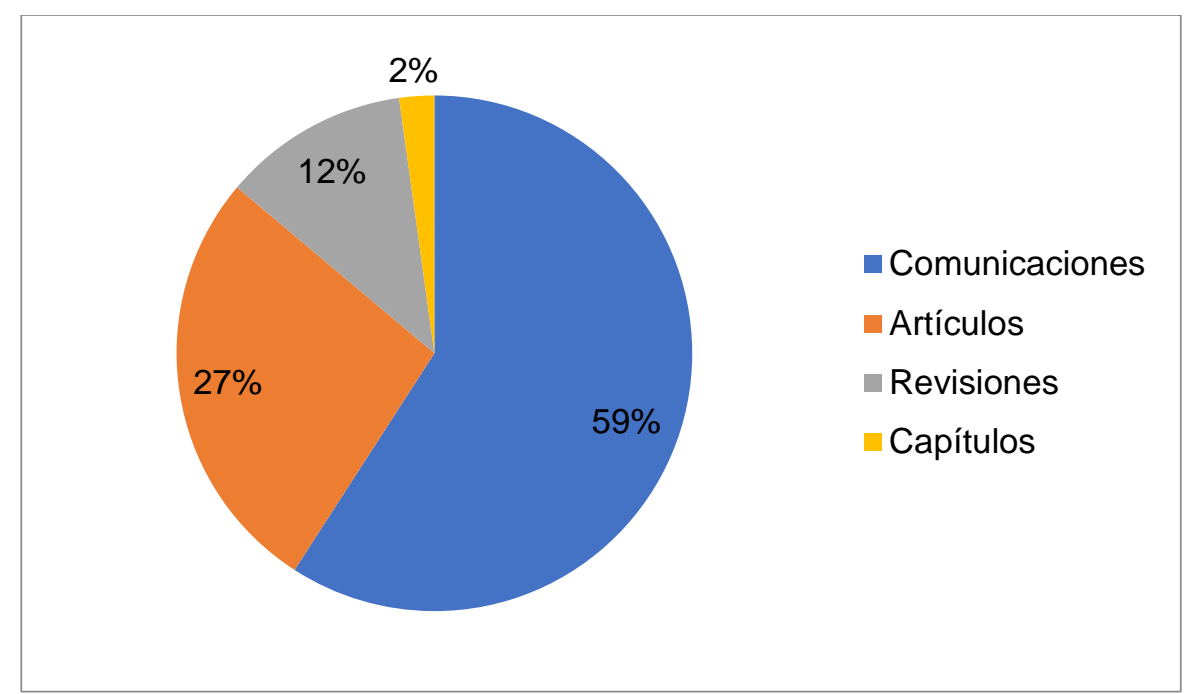

Figura 4. Distribución de los documentos según el tipo de publicación. Fuente: Elaboración propia.

De las 159 palabras clave que aparecen en el total de 137 documentos el término que más se repite es gamification (en 72 documentos) con el mayor grado nodal (53\%). En la Tabla 6 se presentan los 18 términos empleados como palabras clave con una frecuencia de aparición igual o superior a 10. Como se puede observar, las siguientes palabras más recurrentes son education, que aparece en 62 documentos (45\%), seguida de students (30\%), teaching (28\&), e-learning y primary schools (19\% cada una) y edutainment (16\%). Además, se destacan otros términos tan relevantes en la temática como son learning systems (14\%), motivation ( $9 \%)$, serious games $(8,7 \%)$ o educational games $(7 \%)$, entre otros.

Tabla 6

Palabras claves más frecuentes en la producción sobre gamificación en Scopus

Bibliometría aplicada a la gamificación como estrategia digital de aprendizaje. J. Peirats, D. Marín y M.I. Vidal 
RED. Revista de Educación a Distancia. Núm. 60, Artíc. 05, 30-10-2019

DOI: http://dx.doi.org/10.6018/red/60/05

\begin{tabular}{lll}
\hline Palabra clave & $\mathbf{N}$ & $\mathbf{\%} \mathbf{n = 1 3 7}$ \\
\hline Gamification & 72 & $53 \%$ \\
\hline Education & 62 & $45 \%$ \\
\hline Students & 41 & $30 \%$ \\
\hline Teaching & 38 & $28 \%$ \\
\hline E-learning & 26 & $19 \%$ \\
\hline Primary Schools & 26 & $19 \%$ \\
\hline Edutainment & 22 & $16 \%$ \\
\hline Game-based Learning & 20 & $15 \%$ \\
\hline Computer Aided Instruction & 15 & $11 \%$ \\
\hline Computer Games & 14 & $10 \%$ \\
\hline Learning Systems & 14 & $10 \%$ \\
\hline Eotivation & 13 & $9 \%$ \\
\hline Engineering Education & 12 & $8,7 \%$ \\
\hline Human Computer Interaction & 12 & $8,7 \%$ \\
\hline Serious Games & 12 & $8,7 \%$ \\
\hline Curricula & 12 & $8,7 \%$ \\
\hline Educational Game & 11 & $8 \%$ \\
\hline
\end{tabular}

Fuente: Elaboración propia a partir de los datos de Vosviewer

Finalmente, en la Figura 5 observamos la coocurrencia de las palabras clave en los 137 documentos analizados de la base Scopus, que se refiere a las relaciones subyacentes entre los términos (por su tendencia a aparecer juntos) y que nos permite agruparlos en categorías. Se distinguen seis grandes grupos o clusters, pero nos centraremos en los más relevantes; el primero de ellos gira entorno a lo computacional y relaciona términos como: gamification computer programming, design, cooperative learning o interactive learning, entre otros. Por su parte, el segundo clúster se relaciona más con el ámbito educativo e incluye términos como education, curricula, students, teaching, learning, primary schools o edutainment. Por último, el tercero añade términos relacionados con nuevas estrategias o metodologías que relacionan las tecnologías con la enseñanza, tales como: artificial intelligence, mobile learning, e-learning o virtual reality, entre otros. Todos los grupos de palabras coocurren entre ellos por lo que presentan una cercana vinculación conceptual.

Bibliometría aplicada a la gamificación como estrategia digital de aprendizaje. J. Peirats, D. Marín y M.I. Vidal 
RED. Revista de Educación a Distancia. Núm. 60, Artíc. 05, 30-10-2019

DOI: http://dx.doi.org/10.6018/red/60/05

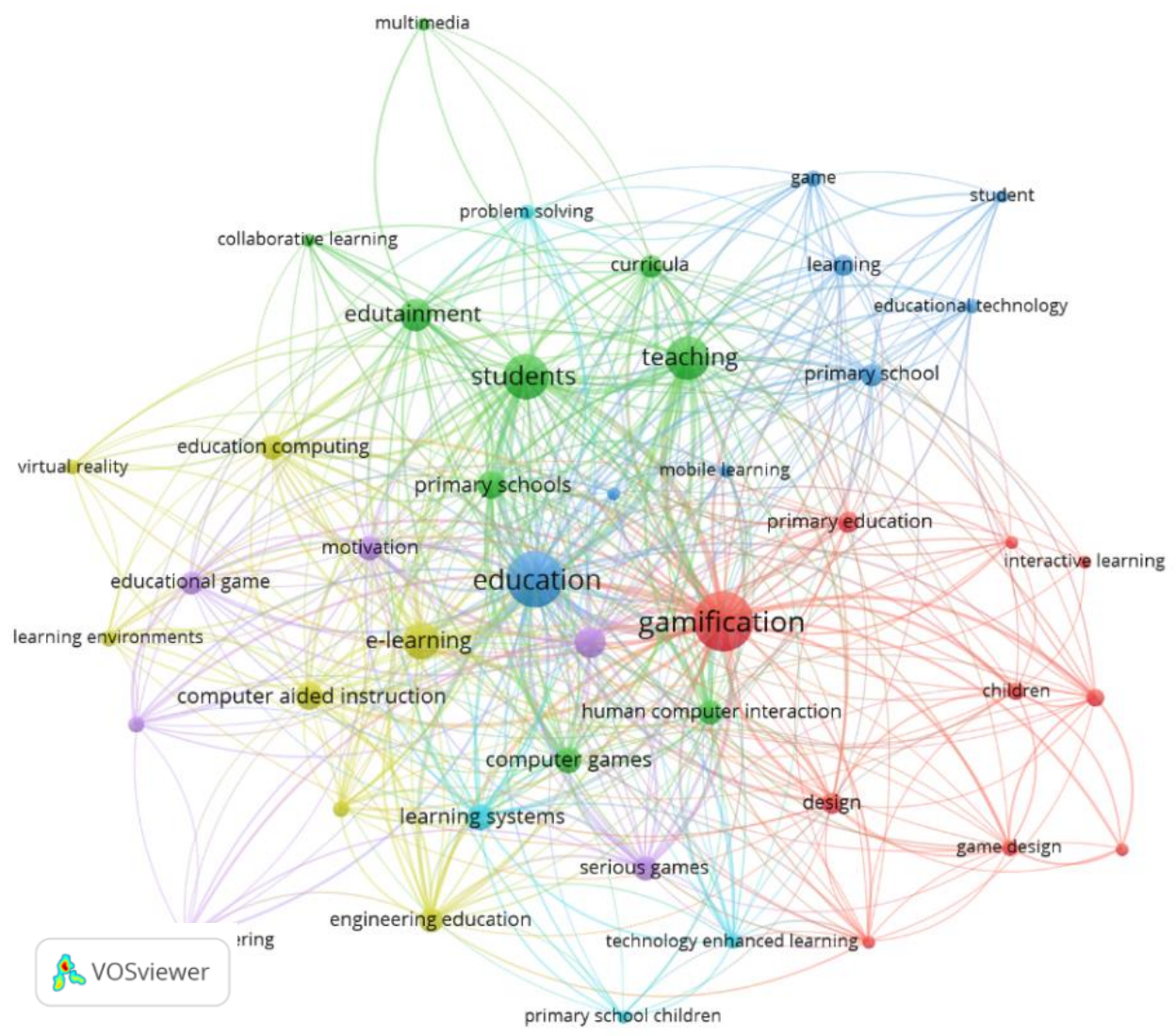

Figura 5. Mapa de coocurrencia de términos. Fuente: VOSviewer

Procediendo con la segunda parte de nuestro estudio, señalamos los principales datos extraídos del análisis de contenido de los 59 documentos del área de Ciencias Sociales. Se tomaron como categorías las etapas educativas y las asignaturas en las que se emplea la gamificación, los dispositivos y las aplicaciones utilizadas, y los principales resultados obtenidos.

Respecto a la primera categoría, las etapas en las que se sitúa la investigación, consideramos interesante destacar que, pese a que nuestra búsqueda incluía como términos clave infantil y primaria, los resultados obtenidos no se corresponden del todo con lo esperado. Por una parte, únicamente se registra un documento en la etapa de infantil, el de Florou, Mavroudi, Haidi, Gouscos y Meimaris (2009), que incluye un estudio de caso en esta etapa, junto con otros dos en primaria y en educación especial; siendo el juego una de las principales herramientas de aprendizaje en la educación infantil, sorprende que apenas se registra un documento publicado sobre el uso de la gamificación en la etapa. Por otra, la mayoría de las publicaciones se refieren a aplicaciones en grupos de primaria (Bratitsis, Meireles \& Neto, 2017; Caldeiro, Yot \& Castro, 2018; García, Jurdi, Jaén \& Nacher, 2018; Oliveira \& Cruz, 2018; Tsarava et al., 2017), aunque también se contemplan siete documentos de secundaria (Cruz \& Orange, 2016; Gifreu, 2015; Isayama, Ishiyama, Relator \& Yamazaki, 2016; Mystakidis \& Berki, 2018; O'Donnell \& Jouy, 2015; Paunova, Terzieva, Dimitrov \& Boneva, 2018; Petrucco \& Agostini, 2016) y quince en estudios superiores (Carrillo et al., 2018; Landicho et al., 2017; Sánchez, Cañada \& Dávila, 2017). Entre estos últimos destacamos las investigaciones llevadas a cabo en los grados de formación de maestros (Cózar \& Sáez, 2016; Sánchez, Cañada \& Dávila, 2018), para ser aplicadas posteriormente en las aulas, o para averiguar las percepciones de los futuros maestros al respecto (Marín, López \& Maldonado, 2015). Al margen de los grados educativos, también se registran algunas en grados científico-técnicos como ingeniería (Assante, Fornario, Sayed \& Salem, 2016) 
o informática (Charles, Hanna, Paul \& Charles, 2012) más centradas en la producción y el desarrollo.

En cuanto a las asignaturas, obtenemos de nuestro análisis que los resultados son variados. Por una parte, la gamificación se emplea para la mejora de la competencia lingüística, tanto de la lengua materna (en ortografía: Kekuluthotuwage \& Fernando, 2018; en lectura: Chen, Li, Lee \& Chu, 2018, o Letchumanan, Muthusamy, Govindasamy \& Farashaiyan, 2016: y en escritura: Rinnert, Martens, Mooney, Talbott \& Rinnert, 2017), como de las extranjeras (Cruz \& Orange, 2016; Oliveira \& Cruz, 2018; Ramírez, EIMiligi, Walton \& Billy, 2018; Shitiq \& Mahmud, 2010). Además, en áreas humanísticas y sociales se ha utilizado para asignaturas como humanidades (Chadimova, 2015), historia (Mystakidis \& Berki, 2018; Petrucco \& Agostini, 2016; Zikas et al., 2016) y ciudadanía (Bratitsis, Meireles \& Neto, 2017). Por su parte, también se recurre a la gamificación en áreas científicas y tecnológicas: se registran documentos que trabajan la educación ambiental (O'Donnell \& Jouy, 2015), las STEM (Kintsakis \& Rangoussi, 2017), la ingeniería (Souza et al., 2017), la electrónica (Assante et al., 2016), las ciencias computacionales (Lunn et al., 2016) las matemáticas (Hwa, 2018; Jagušt, Botički \& So, 2018; Ramdane, Souad, Marusin \& Sidek, 2018) y las ciencias naturales (Carrillo et al., 2018; Sánchez, Cañada \& Dávila, 2018). Se registra asimismo un documento en el que se utiliza para actividades extracurriculares (Amin, Rawi, Isa \& Rahim, 2017). Señalamos que, en cuanto a las asignaturas trabajadas, pese a que los resultados son dispares, el peso de las áreas científicas y las humanísticas está equilibrado, registrándose alrededor de un $50 \%$ de los documentos en cada rama. Por su parte es de destacar que no se registran publicaciones que trabajen las asignaturas artísticas.

En referencia al hardware empleado, destacan los dispositivos móviles, entre los cuales subrayamos las tabletas (Nousiainen, Kangas, Rikala \& Vesisenaho, 2018; Halloluwa, Usoof, Hewagamage, 2014), los móviles (Landicho et al., 2017) y el iPad (Rinnert et al., 2017); y otros se centran en el uso de la computadora mediante audio games (Rovithis, Floros \& Kotsira, 2018) o páginas webs (Cruz y Orange, 2016). En cuanto al software, hemos registrado una gran variedad de recursos, entre los que podemos encontrar aplicaciones usadas en plataformas como Moodle (Kintsakis \& Rangoussi, 2017) o estrategias como la realidad virtual (Mystakidis \& Berki, 2018); o los videojuegos (comerciales -Monga, Jain, Kumar \& Sandeep, 2017- o en línea -Bratitsis, Meireles \& Neto, 2017). Además de aplicaciones específicas conocidas como: Energetic Alpha (Rinnert et al., 2017), Learn to Scout (Amin et al., 2017), MinecraftEdu (Cózar \& Sáez, 2016), ClassDojo (Gooch, Vasalou \& Benton, 2016), ClassCraft (Bretherton, Sim \& Read, 2016), e@Leader (Dickinson \& Hui, 2009) o Facebook (Assante et al., 2016), entre otras que muestran la gran variedad existente.

Por último, en cuanto a los resultados obtenidos en los estudios analizados destacan, por una parte, aquellos que concluyen que el uso de los videojuegos y de la gamificación ofrece ventajas para la competencia docente en cuanto a conocimientos sobre aprendizaje basado en juegos (Nousiainen et al., 2018) y sobre estrategias para incrementar la implicación en el aprendizaje (Cózar \& Sáez, 2016). Por otra, se registran documentos que refieren mejoras en la motivación del alumnado (Carrillo et al., 2018; Douadi, Tahar \& Hamid, 2012), en el interés (Shitiq \& Mahmud, 2010), en las actitudes (Hwa, 2018) y en el incremento del atractivo del aprendizaje a través del uso de serious games o e-learning; también en el alumnado con necesidades educativas especiales (Gooch, Vasalou \& Benton, 2016). Del mismo modo encontramos artículos referidos a beneficios en cuanto a educación en valores como mejoras en la colaboración, el respeto (García, Jurdi, Jaén \& Nacher, 2018), el rendimiento emocional (Sánchez, Cañada \& Dávila, 2018; Gennari et al., 2017), el compromiso con las tareas (Zikas et al., 2016), la autoeficacia, la autonomía (Chen et al., 2018) las relaciones familiares y con amigos (Jagušt, Botički \& So, 2018). Asimismo, este conjunto de estrategias es considerado por algunos como una herramienta óptima para transformar contenidos

Bibliometría aplicada a la gamificación como estrategia digital de aprendizaje. J. Peirats, D. Marín y M.I. Vidal 
impopulares en agradables (Mpiladeri, Palaigeorgiou \& Lemonidis, 2016), como las matemáticas (Hwa, 2018) o las habilidades comunicativas (Oliveira \& Cruz, 2018). Finalmente, se encuentran los documentos en los que, tanto el alumnado como el profesorado los elige preferentemente, en perjuicio de los materiales tradicionales, porque se obtienen mejores resultados en el aprendizaje (Fabricio, 2018; Landicho et al., 2017; Letchumanan et al., 2016; Ramírez, EIMiligi, Walton \& Billy, 2018) y porque satisface las necesidades de los niños y jóvenes de hoy (Gifreu, 2015). Por último, solo un documento (Ternblad \& Guiz, 2018) obtiene como resultado que no se registran diferencias entre el aprendizaje a través de material tradicional y recursos digitales $\mathrm{y} / \mathrm{o}$ gamificados.

\section{Discusión y conclusión}

Desde el convencimiento de que en el cambio y la innovación contextualizada en la realidad se halla el éxito, los propósitos de este trabajo han sido: conocer las últimas tendencias en investigación sobre gamificación como estrategia digital en educación; analizar las características fundamentales de las producciones científicas realizadas y poder sentar bases estables, basadas en la evidencia científica, para una adecuada prospección de la intervención y el aprendizaje gamificado.

En base a ello, se ha realizado un análisis bibliométrico de la producción científica depositada en la base de datos Scopus, elegida por su reconocida acreditación entre los investigadores de todo el mundo. Para la indagación se ha tomado como referencia, ajustándonos a las limitaciones de espacio de la revista, una selección de los indicadores de productividad científica, colaboración, consumo y de repercusión e impacto, así como variables estructurales de todos los artículos disponibles; y el análisis de contenido se ha fijado en categorías como las etapas educativas, las asignaturas en las que se emplea la gamificación, los dispositivos y las aplicaciones utilizadas, y los resultados obtenidos.

En cuanto al análisis de los indicadores, los datos muestran que el interés y esfuerzo por el estudio de la gamificación en todas las etapas educativas y desde diversas áreas de conocimiento aumenta de forma progresiva y constante, especialmente en los cinco últimos años. Este interés se refleja fielmente en la cantidad de artículos disponibles en la base de datos estudiada y en la reciente fecha de publicación, un ejemplo puede ser el texto de Rocha, Gomes y De Melo (2016), que en solo dos años acumula 48 citas. A nivel mundial, entre los países con mayor producción científica en este campo se destaca la posición predominante del continente europeo, especialmente los países más meridionales (Italia, España y Grecia, por orden de producción) que sugiere, tal vez, connotaciones relacionadas con el carácter 0 personalidad de estas naciones. De los resultados del análisis de los datos de coautoría de los trabajos publicados se resalta como significativo un alto grado de firmantes, lo que señala un cierto tejido o tendencia de colaboración entre los investigadores interesados en este campo, como muestra se puede indicar el trabajo de Agoritsas et al. (2014) con 13 coautores.

En el área de las Ciencias Sociales, los datos del presente estudio permiten identificar la existencia de líneas estables en la publicación sobre gamificación, como son el mayor número de textos centrados en la etapa de primaria, entre ellos el de Martínez Pérez (2017), aunque aparecen de todas las etapas; el equilibrio de trabajos entre los tradicionales bloques de asignaturas (científicas y humanísticas) y su no existencia en las artísticas, lo que sorprende en principio en una estrategia que se apoya considerablemente en lo visual.

Para ir finalizando se señala que, en esta época de progresiva digitalización y enorme impacto de las tecnologías en todos los ámbitos de la vida en general, y en la escuela en particular, es de destacar que son muchas las posibilidades que nos ofrece la gamificación, como estrategia didáctica, en cualquiera de las distintas etapas

Bibliometría aplicada a la gamificación como estrategia digital de aprendizaje. J. Peirats, D. Marín y M.I. Vidal 
educativas. Cabe remarcar también del estudio realizado que subyacen y complementan a la analizada otras estrategias docentes que permiten otras formas innovadoras de enseñar y aprender en los centros educativos como pueden ser, entre otras, la robótica (Jesse, 2006), la realidad virtual (Mystakidis \& Berki, 2018) o la inteligencia artificial; separadas, combinadas o juntas es innegable que pueden ser unas herramientas excelentes para abordar con decisión el problema del fracaso escolar señalado al principio de estas páginas.

Para la aplicación de esta estrategia didáctica digitalizada en el aula es necesario contar con profesionales de la educación formados, además de la dotación tecnológica y programaciones flexibles que permitan introducir nuevas formas de hacer y de construir el aprendizaje. Porque, en definitiva, una vez más, la clave se encuentra en el profesorado y en situar en el centro del proceso al alumnado.

En cuanto a líneas de investigación prospectiva o interrogantes sin resolver por ahora, sería conveniente reflexionar sobre el presente y futuro de las estrategias educativas en el contexto de la sociedad digital, además de contrastar sistemáticamente resultados de estudios realizados que desvelen beneficios, pero también malas praxis educativas con el objeto de descartar unas y considerar otras. Más concretamente, sería conveniente indagar sobre los efectos de estas nuevas estrategias en el proceso de enseñanza y aprendizaje; o las repercusiones que tienen en la figura del profesorado y en qué medida la están modificando; el papel que asumen o deberían asumir las familias o, finalmente, cómo serán los planes de estudio para los próximos años implementados con estrategias digitales.

Presentación del artículo: 3 de julio de 2019 Fecha de aprobación: 6 de octubre de 2019 Fecha de publicación: 30 de octubre de 2019

Peirats, J., Marín, D. y Vidal, M.I. (2019). Bibliometría aplicada a la gamificación como estrategia digital de aprendizaje. RED. Revista de Educación a Distancia, 60. DOI: http://dx.doi.org/10.6018/red/60/05

\section{Financiación}

Este trabajo es parte del proyecto de tesis doctoral con referencia FPU17/00372 financiado por el Ministerio de Ciencia, Innovación y Universidades.

Los resultados ofrecidos son fruto también del proyecto de investigación: Análisis de las estrategias docentes del profesorado ante la digitalización de los contenidos del curriculum de educación infantil y primaria (GV/2018/074). Financiado por la Conselleria de Educación, Investigación, Cultura y Deporte de la Generalitat Valenciana.

\section{Referencias}

*Agoritsas, T., Iserman, E., Hobson, N., Cohen, N., Cohen, A., Roshanov, P., (...) Haynes, R.B. (2014). Increasing the quantity and quality of searching for current best evidence to answer clinical questions: Protocol and intervention design of the MacPLUS FS factorial randomized controlled trials. Implementation Science, 9(1) doi:10.1186/s13012-014-0125-9

Aguilar, T.A., \& Aguilar, P.A. (2018). La gamificación como estrategia de motivación en el aula. En A. Torres y L. Romero (eds.), Gamificación en Iberoamérica. Experiencias desde la comunicación y la educación (pp. 137-146). Editorial Universitaria Abya-Yala.

Bibliometría aplicada a la gamificación como estrategia digital de aprendizaje. J. Peirats, 
Alamanda, D. T., Anggadwita, G., Ramdhani, A., Putri, M. K., \& Susilawati, W. (2019). Kahoot!: A Game-Based Learning Tool as an Effective Medium to Improve Students' Achievement in Rural Areas. In Opening Up Education for Inclusivity Across Digital Economies and Societies (pp. 191-208). IGI Global.

Aleixandre, R. (2010). Bibliometría e indicadores de producción científica. En J. Jiménez, J.M. Argimon, A. Martín \& T. Vilardell (Eds.), Publicación científica biomédica: cómo escribir y publicar un artículo de investigación (pp. 363-384). Barcelona: Editorial Elsevier.

*Amin, M., Rawi, N., Isa, W., \& Rahim, N. (2017). Heuristic evaluation of usability for gamified co-curricular lesson: A study case of learn to scout. Advanced Science Letters, 23(5), 4197-4201. doi:10.1166/asl.2017.8274

Area, M. \& González, J. (2015). De la enseñanza con libros de texto al aprendizaje en espacios online gamificados. Educatio Siglo XXI, 33(3), 15-38.

Area, M. (2018). Hacia la universidad digital: ¿dónde estamos y a dónde vamos? RIED. Revista Iberoamericana de Educación a Distancia, 21(2), 25-30.

*Assante, D., Fornario, C., Sayed, A.E., \& Salem, S.A. (2016). Edutronics: Gamification for introducing kids to electronics. IEEE Global Engineering Education Conference, EDUCON, 10-13-April-2016, 905-908. doi:10.1109/EDUCON.2016.7474659

Ballesta, J., \& Lozano, J. (2007). Los medios de comunicación ¿nos igualan o nos diferencian? Enseñanza \& Teaching: Revista interuniversitaria de didáctica, 25, 45-67.

Becker, K. (2016). Choosing and using digital games in the classroom. New York: Springer Science and Business Media.

Biel, L., \& García, A. (2015). Gamificar: El uso de los elementos del juego en la enseñanza de español. Actas del Congreso Internacional de la AEPE (Asociación Española de Profesores de Español). La cultura hispánica: de sus orígenes al siglo XXI. (pp. 73-84). Burgos: Universidad de Isabel I de Castilla.

Bona, C. (2016). Las escuelas que cambian el mundo. Barcelona: Plaza Janés.

Bordons, M., \& Zulueta, M. (1999). Evaluación de la actividad científica a través de indicadores bibliométricos. Revista española de cardiología, 52(10), 790-800.

Borrás-Gené, O. (2018, September). Use of digital badges for training in digital skills within higher education. In 2018 International Symposium on Computers in Education (SIIE) (pp. 1-7). IEEE.

Botha, A., \& Herselman, M. (2015, December). Icts in rural education: Let the game begin. In Proceedings of the 2015 Annual Symposium on Computing for Development (pp. 105-113). ACM.

*Bratitsis, T., Meireles, G., \& Neto, C. (2017). WeAreEurope: An online game for european citizenship education for primary school. Paper presented at the Proceedings of the 11th European Conference on Games Based Learning, ECGBL, 38-46.

*Bretherton, W., Sim, G., \& Read, J.C. (2016). ClassCraft in the primary school classroom. Paper presented at the Proceedings of the European Conference on Games-Based Learning, 67-74.

*Caldeiro, M., Yot, C., \& Castro, A. (2018). Detection of mobile devices-based good educational practices in primary school. Prisma Social, 20, 58-75.

*Calderón, A., Trinidad, M., Ruiz, M., \& O'Connor, R.V. (2018). Teaching software processes and standards: A review of serious games approaches doi:10.1007/978-3-030-00623-5_11

${ }^{*}$ Caponetto, I., Earp, J., \& Ott, M. (2014). Gamification and education: A literature review. Paper presented at the Proceedings of the European Conference on GamesBased Learning, 1, 50-57.

Bibliometría aplicada a la gamificación como estrategia digital de aprendizaje. J. Peirats, 
*Carrillo, D., García, A., Laguna, T., Magán, G., Olalla, A., \& Moreno, J. (2018). Game based learning in laboratory practice. Paper presented at the Proceedings of the European Conference on e-Learning, ECEL, 322-328.

Castellón, L. \& Jaramillo, Ó. (2012). Educación y videojuegos: hacia un aprendizaje inmersivo. Homo videoludens, 2, 264-281.

${ }^{*}$ Chadimova, L. (2015). Creation of 3D models of chosen historical buildings for supporting knowledge transfer. Paper presented at the Digital Heritage International Congress, 747-748. doi:10.1109/DigitalHeritage.2015.7419617

${ }^{*}$ Charles, D., Hanna, C., Paul, R., \& Charles, T. (2012). Rapid development of games inspired metacognitive learning experiences using moodle and gamemaker. Paper presented at the Proceedings of the European Conference on GamesBased Learning,

${ }^{*}$ Chen, X., Li, X., Lee, S.Y.C., \& Chu, S.K.W. (2018). Re-examining students' reading experience in a gamified context from a self-determination perspective: $A$ multiple-case study. Proceedings of the Association for Information Science and Technology, 55(1), 66-75. doi:10.1002/pra2.2018.14505501008

Corchuelo, C. A. (2018). Gamificación en educación superior: experiencia innovadora para motivar estudiantes y dinamizar contenidos en el aula. Edutec. Revista Electrónica de Tecnología Educativa, 63, 29-41. doi: 10.21556/edutec.2018.63.927

Cortizo, J. C., Carrero, F.M., Monsalve, B., Velasco, A., Díaz, L.I., \& Pérez, J. (2011). Gamificación y Docencia: Lo que la Universidad tiene que aprender de los Videojuegos. VIII Jornadas Internacionales de Innovación Universitaria: Retos y oportunidades del desarrollo de los nuevos títulos en educación superior.

*Cózar, R., \& Sáez, J.M. (2016). Game-based learning and gamification in initial teacher training in the social sciences: An experiment with MinecraftEdu. International Journal of Educational Technology in Higher Education, 13(1) doi:10.1186/s41239-016-0003-4

${ }^{*}$ Cruz, M., \& Orange, E. (2016). 21st century skills in the teaching of foreign languages at primary and secondary schools. Turkish Online Journal of Educational Technology, 2016, 1-12.

*Da Rocha, L., Gomes, A.S., \& De Melo, I.J. (2016). Effectiveness of gamification in the engagement of students. Computers in Human Behavior, 58, 48-63. doi:10.1016/j.chb.2015.11.021

Díaz, J., \& Troyano, Y. (2013). El potencial de la gamificación aplicado al ámbito educativo. III Jornadas de Innovación Docente. Innovación Educativa: respuesta en tiempos de incertidumbre.

*Di Mascio, T., Gennari, R., Melonio, A., \& Vittorini, P. (2014). Gamify your field studies for learning about your learners. In Methodologies and Intelligent Systems for Technology Enhanced Learning (pp. 135-142). Springer, Cham. doi:10.1007/978-3-319-07698-0_17

*Di Mascio, T., Gennari, R., Melonio, A., \& Vittorini, P. (2013). Play and learn about your learners to early form your TEL design. Paper presented at the CEUR Workshop Proceedings, 1147.

*Dickinson, A. R., \& Hui, D. (2009). Enhancing intelligence, english and math competencies in the classroom via e@Leader integrated online edutainment gaming and assessment. In D. Russell, Cases on collaboration in virtual learning environments: Processes and interactions (pp. 263-283) doi:10.4018/978-160566-878-9.ch017

*Dodero, G., Gennari, R., Melonio, A., \& Torello, S. (2015). "There is no rose without a thorn": An assessment of a game design experience for children. Paper presented at the ACM International Conference Proceeding Series, 28, 10-17. doi:10.1145/2808435.2808436

Bibliometría aplicada a la gamificación como estrategia digital de aprendizaje. J. Peirats, 
*Dodero, G., Gennari, R., Melonio, A., \& Torello, S. (2014). Towards tangible gamified co-design at school: Two studies in primary schools. Paper presented at the $\mathrm{CHI}$ PLAY 2014 - Proceedings of the 2014 Annual Symposium on Computer-Human Interaction in Play, 77-86. doi:10.1145/2658537.2658688

*Douadi, B., Tahar, B., \& Hamid, S. (2012). Smart edutainment game for algorithmic thinking. Paper presented at the Procedia - Social and Behavioral Sciences, 31, 454-458. doi:10.1016/j.sbspro.2011.12.085

*Fabricio, I (2018). Using Gamification in Education: A Systematic Literature Review.Paper Presented at Proceedings of the European Conference on Games-based Learning, 931.

Fernández, F., Rodríguez, N. \& Verdeja, M. (2017). Gamificación y uso de dispositivos móviles: a la búsqueda de estrategias metodológicas para incentivar el aprendizaje de conceptos. Actas del III Congreso Internacional de Educación Mediática y Competencia Digital.

Fernández, J., Prieto, E., Alcaraz, V., Sánchez, A.J., \& Grimaldi, M. (2018). Aprendizajes significativos mediante la Gamificación a partir del Juego de Rol: "Las Aldeas de la Historia". Espiral. Cuadernos del Profesorado, 11(22), 69-78.

Flatla, D. R., Gutwin, C., Nacke, L.E., Bateman, S., \& Mandryk, R.L. (2011). Calibration games: making calibration tasks enjoyable by adding motivating game elements. En Proceedings of the 24th annual ACM symposium on User interface software and technology (pp. 403-412). Santa Barbara, California.

*García, F., Jurdi, S., Jaén, J., \& Nácher, V. (2018). Evaluating a tactile and a tangible multi-tablet gamified quiz system for collaborative learning in primary education. Computers and Education, 123, 65-84. doi:10.1016/j.compedu.2018.04.011

García, R., \& Jiménez, C. (2019). Relación entre repetición de curso, rendimiento académico e igualdad en educación: Las aportaciones de PISA. Revista Educación, Política y Sociedad.

*García, M., e Hijón, R. (2018). How gamification impacts on vocational training students. Lecture Notes in Computer Science (including subseries Lecture Notes in Artificial Intelligence) LNAI, pp. 99-103 doi:10.1007/978-3-319-93846-2_19

Gavira, J. F., Gallego, E. P., Rodríguez, V. A., Oliver, A.J.S. \& Puyana, M. G. (2018). Aprendizajes Significativos mediante la Gamificación a partir del Juego de Rol: "Las Aldeas de la Historia". Espiral. Cuadernos del Profesorado, 11(22), 69-78.

*Gennari, R., Melonio, A., Raccanello, D., Brondino, M., Dodero, G., Pasini, M., \& Torello, S. (2017). Children's emotions and quality of products in participatory game design. International Journal of Human Computer Studies, 101, 45-61. doi:10.1016/j.jjhcs.2017.01.006

*Gennari, R., Melonio, A., \& Torello, S. (2017). Gamified probes for cooperative learning: A case study. Multimedia Tools and Applications, 76(4), 4925-4949. doi:10.1007/s11042-016-3543-7

Ghaban, W., \& Hendley, R. (2019). How Different Personalities Benefit From Gamification. Interacting with Computers. 31(2), 138-153.

*Gifreu, A. (2015). Creation and use of interactive applications as teaching formula in the classroom. Opcion, 31(6), 329-350.

Girard, C., Ecalle, J., \& Magnan, A. (2013). Serious games as new educational tools: how effective are they? A meta-analysis of recent studies. Journal of Computer Assisted Learning, 29(3), 207-219.

*Gloria, A. D., Margarone, M., \& Bracco, F. (2007). Using hypermedia as a support for computer science courses in primary school design and evaluation of a web based multimedia framework for primary school. Paper presented at the Webist 2007 - 3rd International Conference on Web Information Systems and Technologies, Proceedings, SEBEG(EL/-) 518-522.

Bibliometría aplicada a la gamificación como estrategia digital de aprendizaje. J. Peirats, 
Gómez, I.M., \& Ruiz, M. (2017). Análisis sobre nuevas metodologías activas basadas en el ABP y en la Gamificación en los estudios de Máster del Profesorado en Educación Secundaria. En Roig-Vila (coord.), Libro de actas de las XV Jornadas de Redes de Investigación en Docencia Universitaria- REDES 2017.

Gómez, R. y García, A. (2018). Bibliotecas, juegos y gamificación: una tendencia de presente con mucho futuro. Anuario ThinkEPI, 12. 125-135.

*Gooch, D., Vasalou, A., \& Benton, L. (2016). Exploring the use of a gamification platform to support students with dyslexia. Paper presented at the IISA 2015 - 6th International Conference on Information, Intelligence, Systems and Applications, doi:10.1109/IISA.2015.7388001

*Gooch, D., Vasalou, A., Benton, L., \& Khaled, R. (2016). Using gamification to motivate students with dyslexia. Paper presented at the Conference on Human Factors in Computing Systems - Proceedings, 969-980. doi:10.1145/2858036.2858231

Granda, J.I., Alonso, A., García, F., Solano, S., Jiménez, C.A., \& Aleixandre, R. (2013). Ciertas ventajas de Scopus sobre Web of Science en un análisis bibliométrico sobre tabaquismo. Revista española de documentación científica, 36(2), 1-11.

Gros, B. (coord.) (2008). Videojuegos y aprendizaje. Barcelona: Graó.

*Halloluwa, H.K.T.C., Usoof, H., \& Hewagamage, K.P. (2014). Stimulating learners' motivation in primary education in sri lanka - A literature review. International Journal of Emerging Technologies in Learning, 9(1), 47-52. doi:10.3991/ijet.v9i1.2964

Hassan, L., Dias, A., \& Hamari, J. (2019). How motivational feedback increases user's benefits and continued use: A study on gamification, quantified-self and social networking. International Journal of Information Management, 46, 151-162.

Henao, L.A.G., Ballesteros, J.A., \& Holguín, L.F.C. (2019). El papel de la academia en la tensión entre las viejas y las nuevas formas de ser y estar frente a las pantallas. Revista Boletín Redipe, 8(2), 98-118.

Hernández, V., Sans, N., Jové, M. C., \& Reverter, J. (2016). Comparación entre Web of Science y Scopus, estudio bibliométrico de las revistas de anatomía y morfología. International Journal of Morphology, 34(4), 1369-1377.

Huizinga, G. (1968). Homo Ludens. Barcelona: Ediciones MC.

*Hwa, S.P. (2018). Pedagogical change in mathematics learning: Harnessing the power of digital game-based learning. Educational Technology and Society, 21(4), 259276.

*Isayama, D., Ishiyama, M., Relator, R., \& Yamazaki, K. (2016). Computer science education for primary and lower secondary school students: Teaching the concept of automata. ACM Transactions on Computing Education, 17(1), 2-28 doi:10.1145/2940331

Iosup, A., \& Epema, D. (2014, March). An experience report on using gamification in technical higher education. In Proceedings of the 45th ACM technical symposium on Computer science education (pp. 27-32). ACM.

*Jagušt, T., Botički, I., \& So, H. (2018). Examining competitive, collaborative and adaptive gamification in young learners' math learning. Computers and Education, 125, 444-457. doi:10.1016/j.compedu.2018.06.022

*Jesse, N. (2006). Decision making and image processing in robot soccer - the challenge of the FIRA MiroSot league. Information control problems in manufacturing (pp. 185-189) doi:10.1016/B978-008044654-7/50165-5

*Johnson, L., Adams, S., Estrada, V., \& Freeman, A. (2014). NMC Horizon Report: 2014 K-12 Edition. Austin, Texas: The New Media Consortium.

Kapp, K. M. (2012). The gamification of learning and instruction (p. 93). San Francisco: Wiley.

Bibliometría aplicada a la gamificación como estrategia digital de aprendizaje. J. Peirats, 
${ }^{*}$ Kekuluthotuwage, P., \& Fernando, P. (2018). HomeSchool: An interactive educational tool for child education. Paper presented at the 2017 National Information Technology Conference, NITC, 34-39. doi:10.1109/NITC.2017.8285652

Kim, S., Song, K., Lockee, B., \& Burton, J. (2017). Gamification in Learning and Education. Virginia. USA: Springer International Publishing AG.

King, J. (1987). A review of bibliometric and other science indicators and their role in research evaluation. Journal of information science, 13(5), 261-276.

*Kintsakis, D., \& Rangoussi, M. (2017). An early introduction to STEM education: Teaching computer programming principles to 5 th graders through an e-learning platform: A game-based approach. Paper presented at the IEEE Global Engineering Education Conference, EDUCON, 17-23. doi:10.1109/EDUCON.2017.7942816

Labrador, E., \& Villegas, E. (2016). Unir Gamificación y Experiencia de Usuario para Mejorar la Experiencia Docente. Revista Iberoamericana de Educación a Distancia. 19 (2). 125 - 142.

*Landicho, J.A., De la Cerna, A.P.A., Marapao, J.J.G., Balhin, G.P., \& Paid, R.M. (2017). Hortari: A gamification application for engaged teaching and learning in higher education. Journal of E-Learning and Knowledge Society, 13(1), 181-187

Lee, J.J. \& Hammer, J. (2011). Gamification in education: What, how, why bother? Academic Exchange. Quarterly, 15(2), 146.

*Letchumanan, K., Muthusamy, P., Govindasamy, P., \& Farashaiyan, A. (2016). Online interactive activities to learn ramayana epic by primary tamil students. Asian Social Science, 12(5), 201-207. doi:10.5539/ass.v12n5p201

*Liu, W., Cheok, A.D., Mei-Ling, C.L., \& Theng, Y. (2007). Mixed reality classroom learning from entertainment. Paper presented at the ACM International Conference Proceeding Series, 274, 65-72. doi:10.1145/1306813.1306830

López, I., Avello, R., Baute, L.M., \& Vidal, M. (2018). Juegos digitales en la educación superior. Educación Médica Superior, 32(1), 264-276.

López, M., Vidal, M.I., Peirats, J., \& San Martín, Á. (2017). La gamificación: enseñanza de la lectoescritura en dificultades de aprendizaje. Actas del V Congreso Internacional de Videojuegos y Educación (CIVE'17).

López, M., Vidal, M.I., Peirats, J., \& Peirats, A. (2017). Gamificación y atención a la diversidad. Revista Comunicación y Pedagogía, 299-300, 37-45.

Lozano, J., Castillo, I.S., \& Veas, A. (2014). Alumnado con necesidades específicas de apoyo educativo de Educación Secundaria Obligatoria y consumo de las TIC en la Región de Murcia.Etic@net, 14, 57-69.

*Lunn, J., Khalaf, M., Hussain, A.J., Al-Jumeily, D., Pich, A., \& McCarthy, S. (2016). The use of serious gaming for open learning environments. Knowledge Management and E-Learning, 8(1), 39-54.

Manzano, A.P. \& Baeza, J.A. (2018). Gamificación transmedia para la divulgación científica y el fomento de vocaciones procientíficas en adolescentes. Comunicar: Revista científica iberoamericana de comunicación y educación, 55, 93-103.

*Marín, V., López, M., \& Maldonado, G. (2015). Can gamification be introduced within primary classes? Digital Education Review, 27, 55-68.

Marín, D., \& Vidal, M.I. (2019). Estrategias docentes ante la digitalización. Aula de innovación educativa, 280, 12-16.

Marsh, T. (2011). Serious games continuum: between games for purpose and experiential environments for purpose. Entertainment Computing, 2, 61-68.

Martí, V. (2017). Aplicación de herramientas TIC en secundaria: Diseño de un portafolio con video-tutoriales y gamificación. Castellón, Universitat Jaume I.

Martín, M., \& Vílchez, L.F. (2017). Videojuegos, gamificación y reflexiones éticas. Cuadernos de ética en clave cotidiana, 7. Madrid: Editorial Perpetuo Socorro.

Bibliometría aplicada a la gamificación como estrategia digital de aprendizaje. J. Peirats, 
*Martínez, J. (2017). Gamificando el huerto escolar en Educación Primaria. Los super héroes al rescate. Actas del III Congreso Internacional de Educación Mediática y Competencia Digital.

Measles, S. \& Abu-Dawood, S. (2015). Gamification: game-based methods and strategies to increase engagement and motivation within an e-learning environment. Paper presented at Society for Information Technology \& Teacher Education International Conference, Austin, TX.

Ministerio de Educación y Formación Profesional (2018). Datos y Cifras Curso escolar 2018/2019. Secretaría General Técnica.

*Monga, C., Jain, J., Kumar, S., \& Sandeep, A. (2017). Context rich digital games for better learnability in the IT project management context. Paper presented at the CSEDU - Proceedings of the 9th International Conference on Computer Supported Education, 573-581.

Montilla, H. Y. (2016). Aprendizaje de la fotografía por medio del metaverso Second Life (Master's thesis, Universidad de La Sabana).

*Mpiladeri, M., Palaigeorgiou, G., \& Lemonidis, C. (2016). Fractangi: A tangible learning environment for learning about fractions with an interactive number line. Paper presented at the Proceedings of the 13th International Conference on Cognition and Exploratory Learning in the Digital Age, CELDA, 157-164.

*Mystakidis, S., \& Berki, E. (2018). The case of literacy motivation: Playful 3d immersive learning environments and problem-focused education for blended digital storytelling. International Journal of Web-Based Learning and Teaching Technologies, 13(1), 64-79. doi:10.4018/IJWLTT.2018010105

*Nousiainen, T., Kangas, M., Rikala, J., \& Vesisenaho, M. (2018). Teacher competencies in game-based pedagogy. Teaching and Teacher Education, 74, 85-97. doi:10.1016/j.tate.2018.04.012

*O'Donnell, B., \& Jouy, P. (2015). Learning from mistakes: A quiz to drill climate experts. Paper presented at the Proceedings of the European Conference on GamesBased Learning, 749-752.

Oliva, H.A. (2016). La gamificación como estrategia metodológica en el contexto educativo universitario. Realidad y Reflexión, 16(44), 108-118.

*Oliveira, S., \& Cruz, M. (2018). The gamification octalysis framework within the primary english teaching process: The quest for a transformative classroom. Revista Lusofona De Educacao, 41(41), 63-82. doi:10.24140/issn.1645-7250.rle41.04

Ortiz, A. M., Jordán, J., \& Agredal, M. (2018). Gamificación en educación: una panorámica sobre el estado de la cuestión. Educação e Pesquisa, 44, e173773

Ortiz, A., \& Suarez, C.M. (2018). Diseño e implementación de una aplicación web como plataforma complementaria de enseñanza y aprendizaje en el área de programación para estudiantes de desarrollo web (Doctoral dissertation).

Panadero, C.A. (2019). Las consecuencias sociales de las dificultades de aprendizaje en niños y adolescentes Social consequences of learning difficulties in children and teenagers. Revista Internacional de Políticas de Bienestar y Trabajo Social International Welfare Policies and Social Work Journal, 91.

Park, S., \& Kim, S. (2019). A Badge Design Framework for a Gamified Learning Environment: Cases Analysis and Literature Review for Badge Design. JMIR serious games, 7(2), doi:10.2196/14342.

*Paunova, E., Terzieva, V., Dimitrov, S., \& Boneva, Y. (2018). Integration of game-based teaching in bulgarian schools: State of theart. Paper presented at the Proceedings of the European Conference on Games-Based Learning, 516-525.

Pektaş, M., \& Kepceoglu, I. (2019). What do prospective teachers think about educational gamification? Science Education International, 30(1).

Peña, J.B., Fernández, E.A., Kirillof, S., \& Tovar, N. (2011). La simulación y los juegos en línea como herramienta para la inmersión educativa. Revista Etic@net, 9(10).

Bibliometría aplicada a la gamificación como estrategia digital de aprendizaje. J. Peirats, 
Pérez, F. \& Aldás, J. (2019). Indicadores sintéticos de las universidades españolas. Madrid: Fundación BBVA e Ivie.

*Pérez, D., Contero, M., \& Alcañiz, M. (2010). Collaborative development of an augmented reality application for digestive and circulatory systems teaching. Proceedings - 10th IEEE International Conference on Advanced Learning Technologies, ICALT'2010, 173-175. doi:10.1109/ICALT.2010.54

*Pérez, I. J., Rivera, E., \& Delgado, M. (2017). Improvement of healthy lifestyle habits in university students through a gamification approach. Nutricion Hospitalaria, 34(4), 942-951. doi:10.20960/nh.669

Perianes, A., Waltman, L., \& van Eck, N.J. (2016). Constructing bibliometric networks: A comparison between full and fractional counting. Journal of Informetrics, 10(4), 1178-1195.

${ }^{*}$ Petrucco, C., \& Agostini, D. (2016). Teaching our cultural heritage using mobile augmented reality. Journal of E-Learning and Knowledge Society, 12(3), 115128.

Piaget, J. (1956). Motricité, perception et intelligence. Enfance, 9(2), 9-14.

Pintor, P. (2017). Gamificando con Kahoot! en evaluación formativa. Infancia, Educación y Aprendizaje (IEYA), 3(2), 112-117.

Prensky, M. (2010). Nativos e inmigrantes digitales. Madrid: Institution educative SEK

Przybylski, A.K., Rigby, C.S., \& Ryan, R.M. (2010). A motivational model of video game engagement. Review of general psychology, 14(2), 154-166.

Raftopoulos, M. (2014). Towards gamification transparency: A conceptual framework for the development of responsible gamified enterprise systems. Journal of Gaming \& Virtual Worlds, 6(2), 159-178. Doi:10.1386/jgvw.6.2.159_1

Rapp, A., Hopfgartner, F., Hamari, J., Linehan, C., \& Cena, F. (2018) 'Strengthening gamification studies: current trends and future opportunities of gamification research', International Journal of Human-Computer Studies. doi:10.1016/j.jjhcs.2018.11.007

*Ramdane, T., Souad, M., Marusin, R., \& Md. Sidek, S.S. (2018). The usefulness of music as a tool of teaching islamic education: Teachers' perspective. AlShajarah, 267-286.

Ramírez C. (2014). Gamificación: mecánicas de juegos en tu vida personal y profesional. Alfaomega.

*Ramírez, G., ElMiligi, H., Walton, P., \& Billy, C. (2018). Revitalization of an indigenous language: Which is better the teacher or the app? Paper presented at the Proceedings of the International Conference on e-Learning, ICEL, 344-350.

${ }^{*}$ Rinnert, G.C., Martens, M., Mooney, A., Talbott, J.A., \& Rinnert, B. (2017). Energetic alpha, playful handwriting practice for children. Proceedings of the ACM Conference on Interaction Design and Children, 687-691. doi:10.1145/3078072.3091981

Rodríguez, J., \& Martín, F. (2019). Análisis bibliográfico de la gamificación en Educación Física. Revista Iberoamericana de Ciencias de la Actividad Física y el Deporte, 8(1), 97-109. doi: 10.24310/riccafd.2019.v8i1.5770

Rodríguez, F., \& Santiago, R. (2015). Cómo motivar a tu alumnado y mejorar el clima en el aula. La Rioja: Digital-Text.

Roemer, C., \& Rundle-Thiele, S. (2018, July). Assessing written communication readability: Will the message be received? In Conference: "Broadening Cultural Horizons in Social Marketing" (p. 229).

*Rosi, A., Brighenti, F., Finistrella, V., Ingrosso, L., Monti, G., Vanelli, M., ... Scazzina, F. (2016). Giocampus school: A "learning through playing" approach to deliver nutritional education to children. International Journal of Food Sciences and Nutrition, 67(2), 207-215. doi:10.3109/09637486.2016.1144720

Bibliometría aplicada a la gamificación como estrategia digital de aprendizaje. J. Peirats, 
${ }^{*}$ Rosi, A., Dall'Asta, M., Brighenti, F., Del Rio, D., Volta, E., Baroni, I., ... Scazzina, F. (2016). The use of new technologies for nutritional education in primary schools: A pilot study. Public Health, 140, 50-55. doi:10.1016/j.puhe.2016.08.021

Rouse, K. E. (2013). Gamification in science education: The relationship of educational games to motivation and achievement (Doctoral dissertation). University of Southern, Mississippi, EEUU..

${ }^{*}$ Rovithis, E., Floros, A., \& Kotsira, L. (2018). Educational audio gamification: Theory and practice. Paper presented at the Proceedings of the European Conference on eLearning, ECEL, 497-505.

Sánchez, J. (2017). El papel del conectivismo en el long life learning: Los nuevos roles del profesorado y alumnado. Aulas virtuales: fórmulas y prácticas, 557.

Sánchez, P., Alfageme, M., \& Serrano, F. (2010). Aspectos Sociales de los videojuegos, RELATEC, 9(1), 43-52.

*Sánchez, J., Cañada, F., \& Dávila, M.A. (2017). Just a game? gamifying a general science class at university: Collaborative and competitive work implications. Thinking Skills and Creativity, 26, 51-59. doi:10.1016/j.tsc.2017.05.003

*Sánchez, J., Cañada, F., \& Dávila, M.A. (2018). Emotional responses to innovative science teaching methods: Acquiring emotional data in a general science teacher education class. Journal of Technology and Science Education, 8(4), 346-359. doi:10.3926/jotse.408

San Martín, Á., \& Peirats, J. (2018). Controversias en la transición del libro de texto en papel y electrónico a los contenidos digitales. Revista de Educación a Distancia, $56(5), 1-17$.

Schiller, F. (1945): La educación estética del hombre. Madrid, Espasa Calpe.

*Shitiq, H.A.A.H., \& Mahmud, R. (2010). Using an edutainment approach of a snake and ladder game for teaching jawi script. Paper presented at the International Conference on Education and Management Technology, Proceedings, 228-232. doi:10.1109/ICEMT.2010.5657667

Smith, K., \& Abrams, S.S. (2019). Gamification and accessibility. The International Journal of Information and Learning Technology, 36 (2), 04-123, doi: 10.1108/IJILT-06-2018- 0061

*Song, M., \& Zhang, S. (2008). EFM: A model for educational game design. Lecture Notes in Computer Science, 5093, 509-517. doi:10.1007/978-3-540-697367_54

Soto, J. M. M., Vivancos, A.E., \& Ferrer, L.A. (2018). Evaluación de un videojuego educativo de contenido histórico. La opinión de los estudiantes. Revista Latinoamericana de Tecnología Educativa-RELATEC, 17(1).

*Souza, M., Veado, L., Moreira, R., Figueiredo, E., \& Costa, H. (2017). Games for learning: Bridging game-related education methods to software engineering knowledge areas. Proceedings 39th International Conference on Software Engineering: Software Engineering and Education Track, 170-179. doi:10.1109/ICSE-SEET.2017.17

*Sugimoto, M., Kusunoki, F., Inagaki, S., Takatoki, K., \& Yoshikawa, A. (2004). A system for supporting collaborative learning with networked sensing boards. Systems and Computers in Japan, 35(9), 39-50. doi:10.1002/scj.10502

*Ternblad, E., \& Gulz, A. (2018). Visualizing knowledge in the era of instructional software and gamification: Challenges in design, method and practical use. Proceedings of International Conference of the Learning Sciences, ICLS, 3, 1463-1464.

Tomás-Górriz, V., \& Tomás-Casterá, V. (2018). La Bibliometría en la evaluación de la actividad científica. Hospital a Domicilio, 2(4), 145-163.

*Tsarava, K., Moeller, K., Pinkwart, N., Butz, M., Trautwein, U., \& Ninaus, M. (2017). Training computational thinking: Game-based unplugged and plugged-in

Bibliometría aplicada a la gamificación como estrategia digital de aprendizaje. J. Peirats, 
activities in primary school. Paper presented at the Proceedings of the 11th European Conference on Games Based Learning, ECGBL, 687-695.

Tori, R. (2016). Tecnologia e metodologia para uma educação sem distância. EmRedeRevista de Educação a Distância, 2(2), 44-55.

Van Eck, N.J., \& Waltman, L. (2011). Text mining and visualization using VOSviewer. arXiv preprint arXiv:1109.2058.

Vázquez, S., \& López, S. (2016), Escuela, TIC e innovación educativa. Digital Education Review, 30, 248-261.

Vassileva, J. (2012). Motivating participation in social computing. User Modeling and User-Adapted Interaction, 22(1-2), 177-201.

Vivar, P. (2015). Fundamento del juego serio en contextos educativos. Lima, Perú: Universidad Federico Villareal Ediciones.

Werbach, K., \& Hunter, D. (2012). For the win: How game thinking can revolutionize your business. Wharton Digital Press.

Zichermann, G., \& Cunningham, C. (2011). Gamification by Design: Implementing Game Mechanics in Web and Mobile Apps. Sebastopol (California): O’Reilly Media, Inc.

*Zikas, P., Bachlitzanakis, V., Papaefthymiou, M., Kateros, S., Georgiou, S., Lydatakis, N., \& Papagiannakis, G. (2016). Mixed reality serious games and gamification for smart education. Proceedings of the European Conference on Games-Based Learning, 805-812.

* Las referencias marcadas con un asterisco corresponden con las que han sido objeto de análisis de nuestro estudio, tanto el bibliométrico como el de contenido.

Bibliometría aplicada a la gamificación como estrategia digital de aprendizaje. J. Peirats, D. Marín y M.I. Vidal 